\title{
POLÍTICAS PÚBLICAS DE COMUNICACIÓN E INTEGRACIÓN LATINOAMERICANA
}

\author{
PUBLIC POLICIES OF COMMUNICATION AND LATIN AMERICAN INTEGRATION
}

\author{
Susana Sel ${ }^{1}$ \\ Universidad de Buenos Aires, Buenos Aires, Argentina.
}

\begin{abstract}
Resumen: Los actuales procesos de democratización de la comunicación en la región, son resistidos por grupos mediáticos-económicos-y-políticos que no se circunscriben a la creación de opinión y construcción de subjetividades, sino que avanzan, institucionalmente, generando las condiciones necesarias para la desestabilización de las democracias latinoamericanas. Las decisiones soberanas para desactivar la dependencia de estos grupos monopólicos y garantizar el derecho humano a la comunicación requieren de una activa participación regional. La avanzada neoliberal de los actuales gobiernos impopulares (como el reciente caso de Argentina y el golpe blando en Brasil) se propone restituir los privilegios naturalizando las lógicas del capital, criminalizando las prácticas sociales, comunicativas y políticas de las comunidades latinoamericanas.
\end{abstract}

Palabras clave: Políticas Públicas, Comunicación, Integración

\begin{abstract}
The ongoing processes of democratization of communication in the region are fiercely resisted by media-economic-and-political groups, which do no limit themselves to the creation of public opinion and the construction of subjectivities, but instead are moving forward, striking against institutions and setting the stage to destabilize the region. The sovereign decisions to deactivate the dependency of these monopolistic tandems and to guarantee the human right to communication, both need of an active regional participation. The neoliberal actions of current new governments (as the recent case in Argentina and the "soft coup" in Brazil) intend to return those groups privileged conditions by naturalizing the logic of capital, criminalizing the social, communication and political practices of Latin American communities.
\end{abstract}

Key words: Public policies, Communication, Integration

\section{INICIATIVAS NACIONALES DE COMUNICACIÓN}

Pese a las dramáticas consecuencias producidas por las transformaciones del capital y su modo de acumulación, entre fines del siglo XX y comienzos del XXI se generó una creciente resistencia mundial de colectivos y organizaciones que en su diversidad de intereses decidieron acciones contra la hegemonía del mercado en protestas y conflictos sociales. Articulados con movimientos sociales y experiencias comunicacionales, también presentes desde el año 2001 en los Foros Sociales Mundiales (FSM) y los Encuentros Hemisféricos de Luchas contra el Área de Libre Comercio de las Américas (ALCA), propusieron iniciativas para hacer realidad los Derechos de la Comunicación en la región. Durante la realización del I Foro Social de las Américas, realizado en 2004 en Ecuador, las redes continentales de

\footnotetext{
${ }^{1}$ Profesora e investigadora en la Facultad de Ciencias Sociales de la misma Universidad y Profesora Emérita de Flacso Ecuador. E-mail: susansel@gmail.com.
} 
comunicación (ADITAL, ALER, AMARC, APC, ALAI, OCLACC y WACC) aprobaron propuestas de acciones conjuntas en ese sentido. Es remarcable el rol de los movimientos de origen rural, desde el Movimiento de los Trabajadores Sin Tierra (MST) de Brasil, la Confederación de Nacionalidades Indígenas del Ecuador y el Movimiento Zapatista en México, hasta el Movimiento del Chapare Boliviano, que lograría el primer presidente indígena con Evo Morales.

Desde 1999, y teniendo como punto de partida el proceso venezolano, se reformula en la región el rol del Estado, aplicando políticas públicas que intentan revertir los marcos jurídicos existentes que garantizaron impunidad y concentración económica en la etapa neoliberal. Estas nuevas políticas intentan regulaciones que permitan crear las condiciones para garantizar el Derecho a la Comunicación, en formas asociadas a los planteos de las Políticas Nacionales de Comunicación $(\mathrm{PNC})^{2}$ de los años '70. Segun Luis Ramiro Beltrán (1976), las Políticas Nacionales de Comunicación son

[...] un conjunto integrado, explícito y duradero de políticas parciales de comunicación armonizadas en un cuerpo coherente de principios y normas dirigidos a guiar la conducta de las instituciones especializadas en el manejo del proceso general de comunicación en un país. (BELTRÁN, 1976)

La promulgación de la Ley de Responsabilidad Civil en Radio y Televisión en la República Bolivariana de Venezuela (2004), recoge el debate y las acciones sobre democratización, participación y acceso a los medios, en uno de los intentos más serios de remisión a los principios formulados como Políticas Nacionales de Comunicación de los años '70. Este proceso venezolano de reformulación de servicio público con acento en la proyección de experiencias comunitarias, se produce en un complejo contexto de convivencia con los medios masivos en poder de grupos concentrados del capital que monopolizaron el campo de la información en las últimas décadas y resisten las regulaciones. La experiencia de diversos medios comunitarios venezolanos, en más de 200 iniciativas articuladas en redes, radios, televisoras, agencias, cuentan con estrategias de participación diversificadas que, directamente con las comunidades, cubren demandas, proveen información y constituyen un

\footnotetext{
${ }^{2}$ En 1976, en la Conferencia Intergubernamental de Comunicación en Costa Rica, se reconoce el grado de concentración económica y tecnológica de países y empresas así como el grado de dependencia de aquellos que no los poseen, y definen el rol del estado en la formulación de las PNC como garantía de acceso, participación y servicio público.
} 
espacio de diversificación de producción de mensajes. También se inscribe aquí la experiencia venezolana del primer canal televisivo ViVe TV, creado en 2003, planteando el desafío de la convivencia de lo estatal y lo comunitario.

En Brasil, los sucesivos intentos de regulaciones que no llegaron a tratarse en el Congreso, fueron la base por la cual los movimientos sociales impulsaron la Primera Conferência Nacional de Comunicação (Confecom) en 2009, muy resistida por las empresas de comunicación concentradas ya que en ese espacio se plantea el control social sobre los medios y la recreación de empresas estatales. Bajo el lema "Comunicación: medios para la construcción de derechos y de ciudadanía en la era digital", la Conferencia abordó la producción de contenidos, medios de distribución, y ciudadanía, derechos y deberes. Teniendo como base esos debates, actualmente se halla en elaboración una Nueva Ley de Medios que la Presidenta Russef aseguró llegará a sancionarse.

En Argentina se sanciona la Ley 26.522 de Servicios de Comunicación Audiovisual en 2009. Producto de la lucha que desde el año 2004 llevaron adelante más de 300 organizaciones sociales, sindicatos, universidades, nucleadas en la Coalición por una Radiodifusión Democrática, la nueva norma establece límites para las prácticas monopólicas, en sintonía con las legislaciones más avanzadas en la materia. Para el Relator de la Libertad de Expresión de la ONU, es un modelo único en el mundo y un ejemplo por garantizar el acceso de todos los sectores manteniendo el pluralismo y la diversidad en el ejercicio de la libertad de expresión, desde sus contenidos hasta las formas en que fuera debatida (La Rue, 2009). La Ley contempla 3 tipo de prestadores en igualdad de condiciones, 1) medios públicos que incluye al estado nacional, los provinciales, los municipales, las universidades públicas nacionales, los pueblos originarios y la iglesia (contemplado en la Constitución Nacional), 2) medios comerciales privados con fines de lucro que incluye a los grandes conglomerados pero también a las pequeñas y medianas empresas (pymes), y por primera vez se incluye como 3) medios comunitarios privados pero sin fines de lucro que incluyen movimientos sociales y sindicatos, entre otros. Además la norma se propone el desarrollo de mecanismos destinados a la promoción, desconcentración y fomento de la competencia con fines de abaratamiento, democratización y universalización del aprovechamiento de las nuevas tecnologías de la información y la comunicación (art 1). Y para ello arbitra mecanismos de porcentajes de producción federal, que amplían, entre otros, el campo laboral de artistas, músicos, periodistas, entre otros, facilitando la emergencia de la diversidad 
cultural en todo el territorio nacional y desarticulando una producción monopólica asentada en la Capital y Gran Buenos Aires desde la que se irradiaba a todo el país. Los grupos concentrados de la comunicación intentan por todos los medios (políticos, legislativos, judiciales) entorpecer el cumplimiento de esta norma, que marca un hito de democratización y ampliación de derechos en el campo comunicacional.

En Uruguay se promulga la Nueva Ley de Radiodifusión Comunitaria No 18232 en 2007, que habilita por primera vez al espacio comunitario como prestador de servicios de comunicación. Hoy Uruguay se encuentra en vísperas de la presentación al Congreso de una Ley integral de Medios de Comunicación.

En el año 2009 el Congreso sanciona la nueva Constitución del Estado Plurinacional de Bolivia en consideración a las aproximadamente 40 etnias indígenas que habitan en su territorio. En 2011 se aprobó por amplia mayoría la "Ley de Telecomunicaciones, Tecnologías de Información y Comunicación". La nueva legislación otorga el 33\% de las licencias al Estado, el 17\% a organizaciones comunitarias, un 17\% a organizaciones indígenas y el 33\% restante al sector privado. Al mismo tiempo establece como principio el Acceso Universal a las telecomunicaciones y tecnologías de información y comunicación, así como al servicio postal, para todas y todos los habitantes del Estado Plurinacional de Bolivia, en ejercicio de sus derechos, relacionados principalmente a la comunicación, la educación, el acceso al conocimiento, la ciencia, la tecnología y la cultura. También aborda principios de Calidad, Continuidad, Protección del Medio Ambiente e Innovación y Neutralidad tecnológicos (a fin de habilitar la libre adopción de tecnologías). Se aseguran los servicios a través del principio de Plurinacionalidad, que fija que el Estado está conformado por la totalidad de habitantes, naciones y pueblos indígenas originarios campesinos así como las comunidades interculturales y afrobolivianas. A su vez, se asegura la adopción de mecanismos para lograr el acceso a los servicios de sectores con menores ingresos y grupos con necesidades especiales, con calidad y precios accesibles.

En Ecuador en 2007 se crea Ecuador TV - Televisión Pública (ECTV), gracias a una provisión de fondos no-reembolsables de $\$ 5$ millones del Banco de Desarrollo Social y Económico de Venezuela (BANDES). El canal fue establecido al mismo tiempo que la instalación de la Asamblea Constituyente, para que las sesiones pudiesen ser transmitidas en vivo a todo el país. Desde su nacimiento Ecuador TV se caracterizó por ser el canal con la tecnología más avanzada existente en el país, con transmisiones vía satélite de forma libre y 
On Line a través de internet para el mundo; sus 25 repetidoras y el canal finalmente estuvo al aire en 2008. Además se creó en ese año la Radio Pública del Ecuador, emisora propiedad del Estado a cargo de la Secretaría Nacional de Comunicación. Y desde el año 2009, y por mandato de su Constitución, se debatió en la Asamblea Nacional, una nueva Ley de Medios, y aprobada en 2013 para regular integralmente los espacios destinados a la comunicación pública, comercial y comunitaria.

En Paraguay se crea la Televisión Pública a fines de 2011 y que durante 6 meses funcionó renovando ética y estéticamente el panorama mediatico, hasta el golpe que derribó al gobierno de Fernando Lugo. Algo similar ocurrió con la Radio Nacional, si bien por definición pública, creada en los años '40, en general fué más una herramienta eficaz de desinformación y propaganda del partido Colorado, con o sin la dictadura de Stroessner (1954-1989), que contenidos relacionados con medios públicos.

\section{ORGANISMOS REGIONALES}

En mayo de 2001, en el Primer Encuentro de Economía Política de la Comunicación del Mercosur, se elabora una declaración conocida como Carta de Buenos Aires, en la cual los investigadores reunidos fijan los ejes que enmarcan la creación en 2002 de ULEPICC (Unión Latina de Economía Política de la Información, la Comunicación y la Cultura ${ }^{3}$. En ellos se reconoce que las Tecnologías de la Información y la Comunicación (TIC's) han adquirido un papel fundamental en el modo de producción y en todos los sectores de la vida social como expresión de una nueva situación estructural del capitalismo, producto de la crisis de transición del siglo XX al XXI. El desarrollo científico y tecnológico -especialmente en lo referente a las TIC, las biotecnologías y el desarrollo de nuevos materiales- demuestra el extraordinario grado de socialización de la producción y la potencialidad que ha alcanzado el conocimiento humano, lo que abre inmensas posibilidades liberadoras y de bienestar a la sociedad. Pero el sistema económico y de poder que llevó a esos desarrollos impide, al mismo tiempo, que aquellas posibilidades se realicen, profundizando, al contrario, el individualismo, la miseria y la violencia. Los sistemas de comunicación de masas reflejan esa situación y sirven para mantener y perfeccionar los mecanismos de alienación, reduciendo las posibilidades de comunicación democrática y liberadora a un tipo de interactividad en que el sujeto social está cada vez más preso del consumismo y la enajenación, como resultado de la

\footnotetext{
${ }^{3}$ Disponible en: <http://ulepicc.org/wp-content/uploads/CARTA-DE-BUENO-AIRES.pdf $>$, may. 2001. Acceso en 22 jun. 2017.
} 
exacerbación del sistema mercantil. Todos los sectores de la comunicación, apoyándose en la convergencia que propician las TIC's pasan por una brutal concentración y centralización apoyados por el poder estatal, mientras que, por otro lado, ese mismo poder dificulta al extremo la acción de aquellos actores no hegemónicos que podrían representar una alternativa democrática y progresista de organización de los sistemas de comunicación como el movimiento de las radios y televisoras comunitarias y todos los sectores de servicio público, imposibilitados de competir contra los poderosos intereses económicos oligopólicos. Es en ese sentido que se releva el rol de los organismos regionales.

Desde su creación en 1991, el Mercado Común del Sur (MERCOSUR) es una unión aduanera suscripta en el Tratado de Asunción, por la República Argentina, la República Federativa de Brasil, la República del Paraguay ${ }^{4}$ y la República Oriental del Uruguay. Con la incorporación posterior de la República Bolivariana de Venezuela (2012) y el Estado Plurinacional de Bolivia (2015) cuenta en calidad de países asociados a Chile, Colombia, Perú y Ecuador. Mercosur se constituye así como un área económica y una plataforma industrial dinámica y competitiva no sólo en la región. Es el cuarto bloque económico del mundo en importancia y volumen de negocios y la quinta economía mundial, considerando el PBI nominal producido por todo el bloque. Entre sus objetivos de integración, se cuentan la libre circulación de bienes, servicios y factores productivos entre los países; la adopción de una política arancelaria y comercial común; la coordinación de políticas macroeconómicas y el compromiso de los Estados Partes de armonizar sus legislaciones. En este sentido, y teniendo en cuenta que el Mercosur se constituye como la quinta economía mundial, son escasos los avances demostrados en el campo comunicacional, siendo que éste ocupa un lugar central en la estructura socioeconómica mundial actual. Porque ese también es el espacio que ocupan, sobre todo los medios masivos de comunicación, en su incidencia en la construcción de lo social, desde identidades individuales hasta una cosmovisión planetaria más asociada al consumo. De allí la necesidad de un abordaje más activo de integración del campo comunicacional regional. En diciembre de 2003 se crea la Reunión Especializada de Autoridades Cinematográficas y Audiovisuales (RECAM) como un órgano consultor del Mercosur en el área cinematográfica y audiovisual, integrada por las Autoridades gubernamentales de cada país en la materia. Su finalidad es analizar, desarrollar e implementar mecanismos destinados a promover la complementación e integración de dichas

\footnotetext{
${ }^{4}$ La República de Paraguay fue suspendida por la situación institucional producto de la destitución del Presidente Fernando Lugo, desde el 22 de junio de 2012.
} 
industrias en la región, la armonización de políticas públicas del sector, la promoción de la libre circulación de bienes y servicios cinematográficos en la región y la armonización de los aspectos legislativos (RECAM, 2003). La actividad de la RECAM se ha centrado en estudios regionales para preservación del patrimonio audiovisual y en la capacitación regional, ambos con financiamiento de la Unión Europea, así como en el estudio de la legislación comparada y cadena de valor, focalizando en el Programa Mercosur Audiovisual (PMA), y en las Redes de Salas Digitales (RSD), en el interés común de accesibilidad a la obra cinematográfica en la región. El Mercosur actúa como una esfera de integración económico- comercial y sociopolítica.

La Unión de Naciones Suramericanas (Unasur) compuesto por el Mercosur y la CAN (Comunidad Andina) es una comunidad política y económica entre 12 países suramericanos (Argentina, Bolivia, Brasil, Chile, Colombia, Ecuador, Guyana, Paraguay, Perú, Surinam, Uruguay y Venezuela), creada en 2004, en Cuzco (Perú), en la Tercera Cumbre Suramericana, pero cuyo tratado constitutivo recién se concretó en 2008, en Brasilia. Está conformada por un Consejo de Jefas y Jefes de Estado y de Gobierno, un Consejo de Ministras y Ministros de Relaciones Exteriores, un Consejo de Delegadas y Delegados, y una Secretaría General. En la actualidad está compuesto por nueve consejos que son el de Salud, el de Desarrollo Social, el de Infraestructura y Planeamiento, el de Educación, Cultura, Ciencia, Tecnología e Innovación, el del Problema Mundial de las Drogas, el de Defensa, el de Economía y Finanzas, el Energético y el Electoral. El grueso de la actividad de la Unasur se ha concentrado en Defensa, Medio Ambiente y Derechos Humanos. La actividad de Unasur es relevante en la resolución pacífica de conflictos, así com en la consolidación democrática y estabilidad regional. Así lo atestiguan los conflictos golpistas en Bolivia (2008), por el uso de bases en Colombia (2009), entre Colombia y Venezuela (2009), intento de golpe en Ecuador (2010), el golpe de estado en Paraguay (2012) y ultimamente se ha manifestado por Argentina (2015) en su lucha contra la usura y por otros intentos.En 2013, en el contexto del Festival de Cine de Mar del Plata en Argentina, se constituyó una comisión para crear el Consejo de Comunicación Audiovisual, aún pendiente de concreción

La Alianza Bolivariana para los Pueblos de Nuestra América (ALBA), se crea en 2004, con el objetivo de luchar contra la pobreza y la exclusión social en América Latina y el Caribe. Es un proyecto de colaboración y complementación política, social y económica entre ciertos países de América Latina y el Caribe, promovida inicialmente por Cuba y Venezuela 
como contrapartida del ALCA (Área de Libre Comercio de las Américas), impulsada por Estados Unidos. Se propone crear mecanismos que aprovechen las ventajas cooperativas entre las diferentes naciones asociadas para compensar las asimetrías, a través de fondos compensatorios y aplicación del TCP, Tratado de Comercio de los Pueblos. Está compuesto por Antigua y Barbuda, Bolivia, Cuba, Dominica, Ecuador, Granada, Nicaragua, San Cristóbal y Nieves, Santa Lucía, San Vicente y las Granadinas, Surinam y Venezuela. Haití figura como miembro especial y Honduras fue expulsada en 2009, por el golpe de estado a Manuel Zelaya. El ALBA tiene una moneda especial, el sucre, también un Banco del Alba, se crearon programas de lucha contra el analfabetismo y el Ecoalba sobre medio ambiente. En 2013 se lanza el canal ALBA TV - Canal en Movimiento, constituyendo el Primer Canal Público Participativo, en la Televisión Digital Abierta de Venezuela, con una programación educativa, cultural e informativa diversa.

La Comunidad de Estados Latinoamericanos y Caribeños (CELAC), creada en 2010 en México pero constituída definitivamente en Venezuela en 2011, es un organismo intergubernamental, heredero del Grupo de Río, que promueve la integración de América Latina y el Caribe. Su conformación por parte de los jefes de Estado lo plantearon como el espacio regional propio que una a los 33 estados.Tal como se desarrollan estas políticas, el grado de articulación regional en el campo comunicacional queda más limitado a formas de colaboración binacionales, como en el caso de la ayuda económica de la República Bolivariana de Venezuela a la República del Ecuador para la instalación de su TV Pública, ó de transferencia de experiencias como es el caso de Argentina, con mayor trayectoria de medios públicos, hacia Ecuador ó Paraguay.

\section{INTEGRACIÓN EN INICIATIVAS COLECTIVAS}

Sin embargo, algunos pasos en la integración se dieron en función de iniciativas colectivas más que políticas generadas desde los organismos regionales, entre ellos podemos destacar:La emergencia de la Televisora del Sur (TELESUR) que desde 2005 y bajo la consigna Nuestro Norte es el Sur iniciara sus transmisiones de aire gratuitas para los países latinoamericanos a través de un satélite ubicado en Caracas. Esta sociedad multiestatal cuenta como socios a Venezuela con el 51\% del capital, Argentina con el 20\%, Cuba con el 19\% y Uruguay con el 10\%. Tiene un Consejo Asesor integrado por 36 personalidades de toda la región. Durante las 24 horas transmite en directo información y producción audiovisual documental recibida de corresponsales mundiales. La Cámara de Representantes del 
Congreso de EE.UU. presentó en 2005 una enmienda que autoriza potenciar transmisiones de radio y televisión hacia Venezuela para contrarrestar el supuesto antiamericanismo de Telesur. Su proponente, el representante republicano por Florida Connie Mack, justificó la medida para impedir la retórica antiamericana que asociaría al medio con Al-Jazzera ${ }^{5}$. Telesur aparece como alternativa al cúmulo de (des)información, publicidad y banalización que se envía diariamente por 3.500 canales de cable y es repetido por los medios locales. El proyecto intenta discutir lo alternativo en tanto marginal y se plantea como masivo, en una propuesta comunicacional de calidad y mejor contenido que la que se enfrenta. Por ello requiere de recursos económicos e instalaciones técnicas que pocos medios podrían permitirse sin el apoyo estatal.En el mismo sentido, la construcción de la Radio del Sur en el año 2010, desde su sede en Venezuela y extendida a la región. Se plantea como la plataforma de apoyo comunicacional del proceso de creación del nuevo sistema multipolar internacional, un instrumento basado en los principios de independencia, igualdad, libre determinación y no intervención en los asuntos internos, solución pacífica de conflictos, cooperación, respeto de los derechos humanos y solidaridad hacia la emancipación y bienestar de la humanidad.

La creación de la Unión Latinoamericana de Agencias de Noticias (ULAN), el 2 de junio de 2011 en Caracas, Venezuela, como asociación supranacional de agencias públicas $\mathrm{y}$ estatales de noticias. Los nueve estados fundadores y sus respectivas agencias son: Argentina/Télam; Estado Plurinacional de Bolivia/Agencia Boliviana de Información; Brasil/Agencia Brasil; Cuba/Prensa Latina; Ecuador/Agencia de Noticias del Ecuador y Sudamérica-Andes; Guatemala/Agencia Guatemalteca de Noticias; México/Noticias MéxicoNotimex; Paraguay/ Información Pública Paraguay -IP; Venezuela/Agencia Venezolana de Noticias. ULAN propone promover la libertad de expresión, la democracia, la paz y el entendimiento entre las naciones, y sus productos comunicacionales estarán fundamentados en el rechazo a toda forma de racismo, dependencia económica y cultural.La adopción regional de un mismo estándar digital de transmisión. Por iniciativa de Brasil (2006), Argentina (2006), y Perú (2009), el resto de los países de Sudamérica (salvo Colombia) implementaron el Sistema Brasileño de Televisión Digital Terrestre (SBTVD-TB), basado en el sistema Japonés denominado ISDB-T, norma que garantiza la aplicación de la nueva tecnología televisiva en todo el territorio. Este consenso regional en materia tecnológica no tiene precedentes y posiciona a Latinoamérica como la región del planeta con el estándar más

\footnotetext{
${ }^{5}$ Disponible en: <http://www.dw.com/es/telesur-medio-de-integracion-o-de-proselitismo/a-1658730 >, jul. 2005. Acceso en 22 jun. 2017.
} 
avanzado de Televisión Digital Terrestre, al utilizar el sistema de modulación OFDM, con el sistema de compresión de video MPEG-4, que mejora el MPEG-2 de uso europeo y japonés ${ }^{6}$ Incluso varios países de África están evaluando la adopción del mismo estándar, por lo que podría consolidarse como un estándar sur-sur.

\section{La creación y lanzamiento de satélites telecomunicacionales en la región.} Contando con la colaboración de China y Rusia, América Latina ingresa a la era espacial, en una era de información globalizada y revelaciones de espionaje, estas iniciativas soberanas representan también procesos autonómicos. Venezuela con sus satélites Bolívar (2008) y Miranda (2012), Ecuador con su Pegaso (2011), Bolivia con su Túpac Katari (2013), Argentina con los Arsat I (2014) y Arsat II (2015), Uruguay con su Antelsat (2014) y Brasil con su SGDC (2015) primer satelite gubernamental de comunicaciones. Estos desarrollos tecnológicos nacionales son trascendentes en terminos de soberanía comunicacional, en una region que segun la CEPAL (2015) posee una desigualdad tecnológica interna, focalizando en el ejemplo del escaso acceso a internet particularmente en Haití, Surinam, Honduras, Paraguay.

\section{PERSPECTIVAS}

Sería impensable analizar estos avances en otros contextos politicos. Avances regulatorios que se verifican en la participación comunitaria y sin fines de lucro en Venezuela (2004), Uruguay (2007), Argentina (2009), Bolivia (2011), Ecuador (2013). El rol actual de los medios de comunicación y sobre todo en aquellos países donde los gobiernos populares han avanzado en estas políticas de ampliación de derechos, se constata su conversión prácticamente en un partido opositor cuyo único objetivo es destituír a los gobiernos a fin de preservar los privilegios que lograron consolidar en complicidad con los regímenes neoliberales que asolaron la región.

Con formas de campañas destituyentes suman, entre otros, a la Sociedad Interamericana de Prensa (SIP) ${ }^{7}$ cámara empresarial de los grandes grupos que funciona cual

\footnotetext{
6 Video explicativo SATVD-T sobre Televisión Digital Terrestre, disponible en: <https://www.youtube.com/watch?v=ElB9PNLYQw8>. Acceso en 22 jun. 2017.

7 Sobre la historia de la SIP consultar el artículo de Yaifred Ron, disponible en: <http://www.rebelion.org/noticia.php?id=94256. Acceso en: 22 jun. 2017
} 
organismo internacional, financiada por las agencias de inteligencia y cumpliendo tareas de desestabilización de los regímenes democráticos. A este fuerte actor social asociado a las cámaras empresariales del sector concentrado, se suman varias de sus representaciones nacionales, así como las redes transnacionales de thinks tanks, presentadas como nucleamientos de intelectuales, que en realidad constituyen fundaciones privadas donde convergen empresarios, dirigentes políticos, economistas, periodistas, y profesionales dedicadas a la producción y promoción de ideas neoliberales en América Latina. Una de esas redes es la Fundación Internacional Libertad (FIL), con sede en Madrid, pero con una base en la ciudad de Rosario, Argentina, dirigida por el escritor Mario Vargas Llosa ${ }^{8}$. Encubiertas en perfiles pseudoacadémicas, se proponen una reorganización continental de las fuerzas neoliberales y la descalificación de los regímenes neodesarrollistas o populistas latinoamericanos, vigentes desde este siglo.

Otros actores políticos involucran desde el poder legislativo a los magistrados del sistema judicial, tal como vimos en el golpe de estado en Paraguay, Honduras o el intento actual en Brasil. Los mismos intereses, disfrazados de empresarios, intelectuales, diputados, senadores ó jueces, en Argentina logran frenar una de las normas más amplias, producto de la consulta y el debate extendidos a toda la sociedad. Ley en cuya elaboración participaron los más destacados especialistas de las universidades públicas, y que contó con una voluntad política que superó las innumerables trabas de los intereses concentrados. En Argentina, el neoliberalismo gobernante desde diciembre 2015 arrasa hoy estos logros.

De allí que la democratización de la comunicación no puede agotarse en sí misma, aun reconociendo que son los propietarios de los medios quienes deciden protagonistas, mensajes, crean opinión, construyen subjetividades, inciden en la forma de vida y propagan odios. En la articulación de los grupos mediáticos con los procesos eleccionarios y con los golpes de estado, duros o blandos, el poder han exhibido su accionar. Si la intervención estatal es imprescindible para garantizar igualdad en el acceso, mayor pluralidad y diversidad cultural, no se puede desconocer la magnitud del enfrentamiento en el proceso de democratización de

\footnotetext{
${ }^{8}$ Lo acompañan personajes internacionales de derecha, entre los que se destaca Marcel Granier del golpista multimedio venezolano RCTV, José M.Aznar de España, Roger Noriega de EEUU, Vicente Fox, Jorge Castañeda de México y Julio Sanguinetti de Uruguay. Cuenta también con notorios dueños de medios y políticos de la derecha argentina como el actual presidente Mauricio Macri, Ricardo López Murphy, y el escritor Marcos Aguinis contra la nueva ley de medios. Disponible en: <http://libertad.org.ar/web/quienessomos.php> y <https://www.pagina12.com.ar/diario/elpais/1-101207-2008-03-24.html〉. Acceso en 22 jun. 2017
} 
la comunicación. Para Adalid Contreras (2015), el sentido pragmático de la integración cobra particular importancia en estos tiempos de posibilidades de articulación entre países y regiones en las características del regionalismo latinoamericano de nueva generación. Y por ello, se requiere profundizar las transformaciones de todos los países, en una activa integración regional.

\section{REFERENCIAS}

ALBA. Cumbres de la Alianza Bolivariana para los Pueblos de América (ALBA). Disponible en: $<$ http://www.portalalba.org/index.php?option=com_content $\&$ view=article\&id=1333:declaraci ones-y-resoluciones-de-las-cumbres-del-alba-tcp\&catid=130\&Itemid=146>.

Acceso en: 22 jun. 2017.

AAVV. La Integración Regional y los Medios de Comunicación: Implicaciones y Desafíos. Montevideo: Centro de Formación para la Integración Regional, 1995.

BELTRÁN, Luis Ramiro. Políticas Nacionales de Comunicación en América Latina: Los primeros pasos. Nueva Sociedad Nro.25. Costa Rica: Fund Nueva Sociedad, 1976.

BYRNE BONFANTIN, Tomás. Las Tareas de la Unión Latinoamericana de Agencias de Noticias (ULAN). Aldea Mundo vol.16. Táchira: Universidad de los Andes, 2011

Disponible en: 〈http://.redalyc.org/articulo.oa?id=54328502005>. Acceso en: 22 jun. 2017.

CARPINETA, María Laura; PERTOT, Werner. Dios los cría y ellos se juntan en Rosario. Disponible en: <https://www.pagina12.com.ar/diario/elpais/1-101207-2008-03-24.html>. Acceso en 22 jun 2017.

CELAC. Cumbres. Disponible en: 〈http://www.sela.org/celac/cumbres〉. Acceso en: 22 jun. 2017.

CEPAL. Estado de la banda ancha en América Latina y el Caribe. Santiago de Chile: Comisión Económica para América Latina y el Caribe (CEPAL) ONU, 2015. Disponible en: $<$ http://repositorio.cepal.org/bitstream/handle/11362/38605/S1500568_es.pdf >. Acceso en: 22 jun. 2017.

CONTRERAS BASPIÑERA, Adalid. El límite es el infinito: Relaciones entre integración y comunicación. Quito: CIESPAL-UASB, 2015.

DW. TeleSUR: ¿medio de integración o de proselitismo?. Disponible en: $<$ http://www.dw.com/es/telesur-medio-de-integracion-o-de-proselitismo/a-1658730>. Acceso en 22 jun. 2017.

FUNDACIÓN LIBERTAD. Quiénes somos. Disponible en: <http://libertad.org.ar/web/quienes-somos.php>. Acceso en 22 jun. 2017. 
ENCUENTRO DE ECONOMÍA POLÍTICA DE LA COMUNICACIÓN DEL

MERCOSUR. Carta de Bueno Aires. Disponible en: $\langle$ http://ulepicc.org/wpcontent/uploads/CARTA-DE-BUENO-AIRES.pdf $>$. Acceso en 22 jun. 2017

GRANDIO, Mateo. SATVD-T TDT en Argentina Video Explicativo. Disponible en: <https://www.youtube.com/watch?v=ElB9PNLYQw8 >. Acceso en 22 jun. 2017.

RECAM. Creación de la Reunión Especializada de Autoridades Cinematográficas y Audiovisuales del Mercosur (RECAM). Disponible <http://www.recam.org/files/documents/gmc_creacionrecam.pdf $>$. Acceso en: 22 jun. 2017

RON, Yaifred. Los amos de la Sociedad Interamericana de Prensa. Disponible en: < http://www.rebelion.org/noticia.php?id=94256>. Acceso en: 22 jun. 2017.

SEL, Susana y GASLOLI, Pablo. Law No 26,522 of Audiovisual Communication Services: Cultural practices, power and communication. In. MARTENS, C.; MCCHESNEY, Robert W.; VIVARES, Ernesto. The International Political Economy of Communication: Media and Power in South America. London: Palgrave Books, 2014.

UNASUR. Tratado Constitutivo de la Unión de Naciones Suramericanas. Quito, Unasur, 2011. Disponible en: <http://www.unasursg.org/images/descargas/DOCUMENTOS\%20CONSTITUTIVOS\%20D E\%20UNASUR/Tratado-UNASUR-solo.pdf $>$. Acceso en: 22 jun. 2017

Recebido em 03/07/2016. Aceito em 22/03/2017. Publicado em 21/09/2017.

DOI: 10.11606/issn.1676-6288.prolam.2016.117164 